\title{
High quality development path of Chinese sauce-flavor Baijiu industry-Analysis based on consumption data
}

\author{
Cong Peng ${ }^{1, *}$, and $\mathrm{Xu} \mathrm{Guo}^{1}$ \\ ${ }^{1}$ School of Economics and Finance, Guizhou University of Commerce, Guiyang, Guizhou, China, 550014
}

\begin{abstract}
As an important part of Baijiu industry in China, Chinese sauce-flavor Baijiu is sought after by the consumer market for its unique taste. However, in recent years, there are still many challenges in the process of industrial development. Based on the analysis of three characteristics of Chinese sauceflavor Baijiu market and the internal and external environments of industrial development, the internal and external factors of high quality and sustainable development of Chinese sauce-flavor Baijiu industry were discussed from three aspects - concentrated development, green development and diversified development.
\end{abstract}

\section{Introduction}

Baijiu brewing in China has a long time history dated back to ancient times. In the course of evolution, kinds of brewing technology have been created and a unique liquor culture was formed. The custom of raising a cup of liquor has rooted in Chinese culture for hundreds of years. Like French wine and German beer, feverish spirit is one part of people's daily life. No matter it is a commercial banquet, grand celebration, family gathering or friend party, Baijiu (liquor) is always at presence. As one of the important branches of Baijiu industry, Baijiu of sauce-flavor type is mainly produced in the Maotai Town and Renhuai County of Guizhou province, Chishui River Basin, as well as Sichuan and Hunan provinces. At present high-end consumption of Baijiu, the sauce-flavor type occupied half of the Baijiu industry. As early as 1915, Maotai liquor of Guizhou represented China to participate in the "Panama World Exposition" and won the gold medal, becoming one of the "world famous liquors" equaling Scotch whisky of UK and Cognac brandy of France. Since 2001 when Maotai became the state banquet of Zhongnanhai, the seat of CPC, its brand is more well-known in both China and overseas. In 2012 when the Baijiu industry was undergoing an adjustment period, the sauce-flavor Baijiu enterprises deeply realized the market crisis, and formed mission groups to the key Baijiu consumption regions (Beijing, Wuhai, and Zhengzhou) for cultural exchange and promotion of the sauce-flavor Baijiu. By taking a series of measures such as utilizing the advantage of the brand and focusing on superior resources to promote the liquor, the consumers' cognition and acceptance on the sauce-flavor Baijiu were effectively improved. Hence the sauce-flavor Baijiu industry takes on a strong development trend. Whatever in production capacity, market value or in revenues, profits and taxes, the sauce-flavor Baijiu industry has made steady development ${ }^{[1]}$. In 2019, with the production of 400,000 kilo-litre (about $5.1 \%$ of total China's production), the such type of enterprises accomplished the production value of 134.1 billion yuan, $2 \%$ of the total China's Baijiu industry value, among which the sauce-flavor Baijiu enterprises of Guizhou province produced $80 \%$ of the total China's amount, accomplished the production value of 113.1 billion yuan, of which the value of Renhuai city reached 86.9 billion yuan. Affected by the new coronavirus epidemic, the output of sauce-flavor Baijiu decreased $2 \%$ in China. In addition, the price of its production factors such as raw materials raised and the market demand for this type of Baijiu sharply increased, leading to an average increase of $20 \%$ for it. Under the trend of duration of the epidemic, how to deal with new challenges, steadily improve its production, quality and market value, and continue to lead China's Baijiu industry to a sustainable, leap-forward development, it is a thoughtful matter for the sauce-flavor Baijiu enterprises.

\section{Market characteristics of sauce-flavor Baijiu}

In terms of market position, sauce-flavor Baijiu mainly aims at high-end Baijiu (Its market price is higher than RMB 700 yuan.) market. The data of UBS Securities and Forward Industry Research Institute showed that at the high-end Baijiu market in China, 55\% Baijiu is used for family consumption, $43 \%$ for business purpose, and $2 \%$ for government affairs.

From the perspective of consumption ability and age grade among the high-end consumer groups of sauceflavor Baijiu at present, the people aged between 30 and 59 are the major force. Therefore, like other

*Corresponding author: penougs@163.com 
consumption area, people are more pleased to spend more money on high-end Baijiu as consumers become richer. This is one important factor affecting the highend Baijiu market. In addition, China is undergoing an aging period and is also one of the few countries where the consumption on alcohols increases as people get older. Hence it can be predicted that the consumption ability on high-end sauce-flavor Baijiu in the future shows an upward trend.

According to the data of China's Baijiu industry survey by Euromonitor in 2018, the top 5 Baijiu enterprises exceeded $20 \%$ of the whole industry in market share for three successive years, and this trend is still strengthening. Also the research conducted by HSBC $^{[2]}$ revealed that Baijiu output poses a positive correlation relationship with the fixed assets in the whole society, with the correlation coefficient of 0.8 , and there exits a one-year lag relationship between them. Therefore, it is anticipated that in the process of expediting urbanization and with the constant increase of fixed assets, the production and demand of Baijiu will also demonstrate an accelerated rising trend.

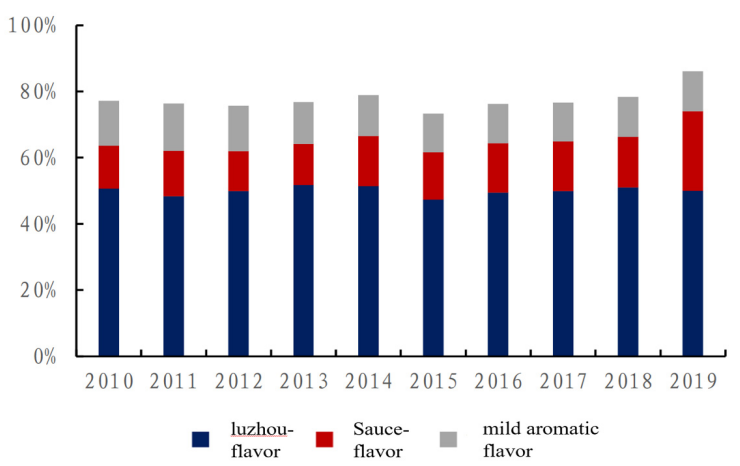

Fig. 1. Proportion of Each Flavour Type

Similar to other luxuries, the supply of high-end sauce-flavor Baijiu is constrained by production technology and cycle, and it is hard to appear a big increase in a short period. Thus a rationing supply in the whole country is inevitably to emerge in the marketing process. Taking Feitian series Baijiu of Guizhou Maotai Group for example, due to the limitation of production capacity, the situation is that it is hard to purchase Feitian Baijiu in the market. As the most accepted sauceflavor Baijiu in the market, Maotai Group has the power to price its products. Feitian series alone occupy $86 \%$ of the group's total revenue and nearly $60 \%$ share in the high-end market, and this rationing shows an upward trend.

\section{The environment of sauce-flavor Baijiu industry}

\subsection{Internal environment}

\subsubsection{Production cost}

Due to the influence of the complex technology, low yield and other factors, the production cost of brewing sauce-flavor Baijiu is much higher than that of other flavor typed ones. Therefore, cost control is the important link in the whole process of sauce-flavor Baijiu brewing. To realize the scale effect of production and operation of the sauce-flavor Baijiu, it not only requires to control every production element, technological process and business link, but also to actively explore how to optimize and ameliorate the business processes. Furthermore, this process should be carried through all the production and operation links such as raw material purchase, starter propagation, brewing, package technology, package design, marketing, logistics transport, pre-sale and after-sale services, and product research and development, etc ${ }^{[3]}$. Firstly, the production cycle of sauce-flavor Baijiu is relatively long, for instance, the time from first feeding to Baijiu yield should go through one whole year, and later it needs three years to store the aged liquor. Then after blending technology, it can be come out of the factory, that is, it requires 5 years to complete the whole brewing process. Such being the case, it not only brings about large amount of money occupied, but also reduces the turnover rate of capital, prolongs the cycle and increases the risk of capital reclaiming. Secondly, the brewing of sauce-flavor Baijiu requires five jin of sorghum to yield one jin finished product. This ratio is far lower than that of other flavor typed Baijiu, which is also the causes of high product cost and large demand for capital.

\subsubsection{Internal competition}

There are many enterprises operating in the whole sauceflavor Baijiu industry. According to incomplete statistics, about 1500 enterprises are seating in the Guizhou province, and their competition in the industry is increasingly fierce. In the industrial pattern of sauceflavor Baijiu in China, large enterprises, especially large state-owned ones, sluggishly respond to the market due to the restriction and limitation of systems and mechanisms. However, large number of medium and small-sized enterprises not only operate in a flexible way, but also closely correspond to the market, for which they possess certain survival opportunities in the market and become a non-negligible power. Although these small and medium-sized enterprises have positive factors, and become an indispensable link in the sauce-flavor Baijiu industry chain, they also result in market instability, for instance, low-price competition, forged and fake commodities, and other phenomena seriously disrupting the market order. The internal fierce competition limits the development rate of the whole industry. In recent years, the competition has been occurring between Guizhou Jinsha Hui Sha Wine Co., Ltd. And Guizhou Xi Jiu Co. Ltd., Maotai Group, which leads to fierce rivalry in the Guizhou sauce-flavor Baijiu industry. Among other things, Maotai Group is making efforts to permeate in the middle and low end market, thus the competition in the sauce-flavor industry is increasingly fierce(Table $\mathbf{1}$ for details). What is more, the integral development is limited due to its environmental bearing capacity ${ }^{[4]}$. 
Table 1. The situation of mainstream sauce-flavor baijiu brands

\begin{tabular}{|c|c|c|c|c|c|c|c|}
\hline Echelon & Brand & $\begin{array}{c}\text { Production } \\
\text { capacity } \\
(10,000 \\
\text { tons })\end{array}$ & $\begin{array}{c}2019 \\
\text { revenue } \\
\text { (billion } \\
\text { yuan) }\end{array}$ & $\begin{array}{c}\text { Growth } \\
\text { rate }\end{array}$ & $\begin{array}{c}\text { Market } \\
\text { share }\end{array}$ & $\begin{array}{l}\text { Price } \\
\text { range } \\
\text { (yuan) }\end{array}$ & $\begin{array}{c}\text { Scale } \\
\text { (billion } \\
\text { yuan) }\end{array}$ \\
\hline $1 \mathrm{st}$ & $\begin{array}{l}\text { Mao-tai } \\
\text { Feitian }\end{array}$ & 4.99 & 758.02 & $15.80 \%$ & $56.10 \%$ & $\begin{array}{c}1499 \\
-3000\end{array}$ & 500 \\
\hline \multirow{7}{*}{$2 \mathrm{nd}$} & \multirow{4}{*}{ Moutai series } & \multirow{4}{*}{2.51} & \multirow{4}{*}{95.42} & \multirow{4}{*}{$18.10 \%$} & \multirow{4}{*}{$7.10 \%$} & 228 & 40 \\
\hline & & & & & & 498 & 10 \\
\hline & & & & & & 426 & 10 \\
\hline & & & & & & 278 & 10 \\
\hline & XiJiu & $2+$ & 75.8 & $40.70 \%$ & $5.60 \%$ & $407-607$ & 42 \\
\hline & \multirow{2}{*}{ Langjiu } & \multirow{2}{*}{1.8} & \multirow{2}{*}{53} & \multirow{2}{*}{$12.00 \%$} & \multirow{2}{*}{$3.90 \%$} & 419 & 32 \\
\hline & & & & & & 1159 & 21 \\
\hline \multirow{5}{*}{$3 \mathrm{rd}$} & Guotai & 0.53 & 18.88 & $60.50 \%$ & $1.40 \%$ & 549 & 13 \\
\hline & Jinsha & 1.9 & 15.26 & $165 \%$ & $1.10 \%$ & 959 & 6.4 \\
\hline & zhenjiu & 0.46 & $10+$ & $67 \%$ & $0.70 \%$ & 600 & 4 \\
\hline & \multirow{2}{*}{ Diaoyutai } & \multirow{2}{*}{0.3} & \multirow{2}{*}{$10+$} & \multirow{2}{*}{$20-30 \%$} & \multirow{2}{*}{$0.70 \%$} & 588 & - \\
\hline & & & & & & 488 & - \\
\hline \multirow{4}{*}{4 th } & \multirow{2}{*}{ Danquan } & \multirow{2}{*}{1.5} & \multirow{2}{*}{$10+$} & \multirow{2}{*}{$150 \%$} & \multirow{2}{*}{$0.70 \%$} & 240 & - \\
\hline & & & & & & 530 & - \\
\hline & Xiantan & 2 & $10+$ & $10 \%+$ & $0.70 \%$ & 618 & - \\
\hline & YunMen & 0.5 & $5+$ & - & $0.40 \%$ & 528 & - \\
\hline
\end{tabular}

other alcoholic beverage is much increasing, too, thus it

\subsection{External environment}

\subsubsection{Threats from substitutes}

Sauce-flavor Baijiu is one kind of beverage containing high alcohol, and this attribute determines that it is not the sole product for consumers to choose. With the improvement of people's living standards, they pay more attention to health, and this brings about the change in the way of their consumption concept and pattern. Therefore, the competition among substitutes for sauceflavor Baijiu also emerges. Firstly, in the Baijiu genealogy of China, apart from sauce-flavor Baijiu, there are strong-flavor, fen-flavor, rice-flavor, feng-flavor, sesame-flavor, soybean-flavor, special flavor, laobaiganflavor, and compatible-flavor ones, etc. They all belong to the beverage containing high alcohol, and this causes high substitutability between them. Secondly, other beverages such as beer, fruit wine, rice wine, and imported wines also occupy the market. With its unique flavor and low content of alcohol, beer enjoys an absolutely dominated advantage among young consumers; and grape represented by fruit wine is also very popular among youngsters for its unique cultural connotation $^{[5]}$. At business banquets and daily consumption, grape serves as a stand-by alcoholic beverage and occupies a very important position ${ }^{[6]}$. Rice wine is another alcoholic beverage produced by China and favored by some consumers for its low content of alcohol, rich in nutrient elements, and multiple values for health care. While all imported wines belong to distilled liquors and contain high content of alcohol, therefore they also become an important substitute for sauceflavor Baijiu. Thirdly, with abundant categories of goods being available in the market since the 21 st century,

\subsubsection{Government policies}

As China implements licensing system for the production of Baijiu, each link of the production of sauce-flavor Baijiu is limited by the government. For example, every three years the government would check the production licence and stops issuing production licence to those enterprises being unable to reach the standard. The government will also stringently execute approval procedures to limit the number of new entrants of Baijiu production. Apart from production licence, sanitary inspection, quality indexes, environmental protection, and carbon emission, etc. are also strictly supervised and regulated by the government. As a rare resource, Baijiu production licence is a barrier to stop external competitors entering ${ }^{[7]}$.

\section{Exploration on high-quality development path of sauce-flavor Baijiu}

\subsection{A concentrated development path}

Industrial concentration is a phenomenon that enterprises with common characteristics or being complementary concentrate in a particular area around an industry and are closely related to each other. Generally speaking, concentrated effect can be realized through geographical concentration of above-mentioned enterprises on a some industry chain, such as reducing the consumption of raw materials, energies and other resources, realizing intensive configuration of resources, reducing costs and enhancing enterprise competition. At present, industrial concentrated development model mainly includes 
exogenous concentration, regional spontaneous concentration and government-dominated industrial park. Among the three, the exogenous concentration model refers to the practice that a certain enterprise utilizes its regional advantage and policy direction in the course of international industrial transfer to introduce foreign investment and establish a cooperated enterprise; the second one is such a phenomenon that different kinds of enterprises spontaneously concentrate around an industry in their formation and development; and the industrial park model is dominated by government and established in a certain geographical area enjoying many governmental favorable policies for purpose of realizing the development goal of a particular industry. In the process of regional economic development, the last model can be rapidly established to introduce foreign capital or foreign investment under the guarantee of a series of governmental favorable policies, and is widely adopted $^{[8]}$. Nowadays, the development model of sauceflavor Baijiu in China is basically the governmentdominated industrial park, in which land, water, electricity, energy and other production elements are generally stressed for concentrated utilization, mainly embodied in the following comprehensive indexesoverall service efficiency of energy, water resource consumption ratio per unit output value, the utilization rate of land resources per unit area, and the standard conformance rate of wastes. While reducing the production and management costs, the enterprise in the park raises its comprehensive utilization rate of energy and strengthens protection on natural resources and ecological environment.

\subsection{A green development path}

A Green development is to follow the circular economic concept of sustainable development and take the road of low-carbon, environmentally friend, green and sustainable development throughout the entire production and operation of sauce-flavor Baijiu. This requires that the goal of low energy consumption, low pollution, low emissions, high efficacy, high efficiency, and high benefit run throughout the entire process of industrial development, and ultimately a win-win social development form in both economic benefits and ecological environment is achieved by taking low carbon and environmental protection as the development direction, energy conservation and emission reduction as the development method, and core technologies as the development breakthrough. In the brewing process of sauce-flavor Baijiu, the waste distiller grains will have a certain negative impact on the environmental ecosystem. With the capacity of sauce-flavor Baijiu being continuously increased, this impact will put a certain pressure on the local natural environment. Therefore, it is necessary to strengthen the upgrading of core technologies, intensify the protection of the ecological environment and the rational use of resources, reinforce the comprehensive management of ecology, environment and resources, and implement biological engineering projects that place equal emphasis on comprehensive pollution control and cleaner production to realize the comprehensive recycling and utilization of brewed fertilizers; in terms of selection and use of energy, we should fully utilized the clean energy such as natural gas, biogas, and clean coal, etc. and fully recover the waste heat and excess heat to reduce energy consumption.

\subsection{A diversified development path}

Baijiu in China has a long history and rich cultural connotations. In the evolution of sauce-flavor Baijiu industry, therefore, this historical trait must always be run though and corresponding cultural (tourism) propagation be carried out. We should take multiple measures such as implementing a concentric and diversified development strategy and focusing on the development of Baijiu industry to cultivate a healthy and favourable wine culture, and ultimately realize the integrated development of the wine industry, cultural and creative industries, transportation and tourism industries.

Taking Renhuai county of Guizhou as an example, Renhuai has unique resource advantages in developing wine culture tourism. Thanks to its long wine-making history, rich wine cultural heritage and unique winemaking technology, the wine industry in Renhuai is very well developed with numerous famous wine brands, among which, Moutai is world-famous, and the construction of wine culture and tourism landscape has also begun to take effect. Therefore, we should integrate tourism resources with wine culture as the core, thoroughly dig the connotation of wine culture and enrich the development of wine culture tourism products; carry out eco-tourism and incorporate the production bases of wine raw materials into the tourism development system; and develop industrial tourism and decrypt the mystery of sauce-flavor Baijiu brewing. Furthermore, we should build Moutai town into a famous, cultural tourism town and achieve new breakthrough in wine tourism of Guizhou; make the "Water Sacrifice Ceremony" during Double Ninth Festival as an important part of wine culture tourism; develop wine cultural heritage tourism and innovate tourism development models. By such ways, the tourism of Guizhou can be driven along with its industry and culture, and in turn to boost sustainable, rapid and healthy development of industries along with its tourism and culture.

\section{Conclusion}

Due to mutual influence and restriction of various factors such as economies of scale, product differentiation, fund demand, conversion cost, control of channels, government policies, competition and counterattack of original enterprises, etc., the new entrants won't bring too much actual threat to the competition of current sauce-flavor Baijiu industry. However, influenced by consumption concepts and habits of consumers, sauceflavor Baijiu and other flavored ones are highly substituted and competed by each other. Meanwhile, attention should be paid to the multiple competitions 
resulted from beer, fruit wine, rice wine, and imports, alcopop, medicated wine and health care wine, soft drinks, alcoholic food and other industries. Nowadays some notable features are seen in the competition of sauce-flavor Baijiu enterprises, for example, Moutai outshining others, competition in the industry intensified by many new entrants, competition methods being more flexible in small and medium-sized enterprises, and focus of competition varied with time and environment. In short, as economy develops and market changes, the competition in the sauce-flavor Baijiu industry will become increasingly fierce. For sauce-flavor Baijiu companies, being old or new entrants, only by carefully studying the industrial competition and formulating correct strategies can they capture the market and develop.

\section{Acknowledgment}

Fund : "Business Administration" Provincial Key Support Discipline (Qian Degree Hezi ZDXK [2016] No. 18)

\section{References}

1. Wen Xiao, Zhaojun Liu. Analysis on the Development of Liquor Industry in Renhuai City under the New Economic Norm [J]. Journal of Anshun College, 2016,18(01): 105-108.

2. SONG K. HSBC Global Research: China Baijiu [Z]. Hong Kong: 2018.

3. Qiang Li, etc. The Design of Enterprise of Maotai Liquor Production Planning and Control System [J]. Journal of Tongren University, 2016,18(4): 159-162.

4. Caiting Ji. Comparative Study on Aroma Components of Different Flavor Liquors [J]. Chemical Engineering Design Communication, 2018,44(10): 128-129.

5. Yao Qin, etc. Study on the Development and Status Quo of China's Fruit Wine [J]. China Brewing, 2020, 39 (09): 1-6.

6. Weiye Tian, etc. Evaluation on Grade Classification of Chinese and Foreign Wine Products and Englightment [J]. China Brewing, 2020,39 (10): 220-224.

7. Bin Liao, Ting Wang, Haitian Wang. Evaluation on Sustainable Competitiveness of Baijiu Enterprises [J]. China Brewing, 2019, 38 (07): 200-204.

8. Ying Jiang, etc. Research on Technological Innovation and Industrial Cluster Development of Traditional Maotai-flavor Baijiu Industry in Guizhou Province [J]. Liquor-Making Science \& Technology, 2011 (05): 42-45. 